\section{PSICOLOGIA IBEROAMERICANA}

\section{Psicología lberoamericana}

ISSN: 1405-0943

psicología.iberoamericana@uia.mx

Universidad Iberoamericana, Ciudad de

México

México

Cortina Guzmán, Laura Graciela; Berenzon Gom, Shoshana

Traducción al español y propiedades psicométricas del instrumento Positivity Self Test

Psicología Iberoamericana, vol. 21, núm. 1, enero-junio, 2013, pp. 53-64

Universidad Iberoamericana, Ciudad de México

Distrito Federal, México

Disponible en: http://www.redalyc.org/articulo.oa?id=133929862007

Cómo citar el artículo

- Número completo

- Más información del artículo

- Página de la revista en redalyc.org

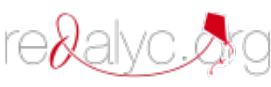

Sistema de Información Científica

Red de Revistas Científicas de América Latina, el Caribe, España y Portugal

Proyecto académico sin fines de lucro, desarrollado bajo la iniciativa de acceso abierto 


\title{
Traducción al español y propiedades psicométricas del instrumento Positivity Self Test
}

\author{
Spanish Translation and Psychometric Properties of the Positivity Self-Test
}

\author{
Laura Graciela Cortina Guzmán* \\ Universidad Nacional Autónoma de México \\ Shoshana Berenzon Gom \\ instituto Nacional de Psiquiatría
}

\section{RESUMEN}

El propósito del presente trabajo fue traducir, adaptar, validar y determinar la confiabilidad de la auto-prueba de positividad (Fredrickson, 2009) en internet. Para ello, se tradujo el instrumento al español, se corroboró la pertinencia cultural mediante laboratorios cognitivos, se probó por escrito y en internet con estudiantes universitarios. Se determinó la estructura factorial y confiabilidad en internet en una muestra no probabilística formada por 383 estudiantes de la Escuela de Enfermería y Obstetricia de la UnAm. El análisis mostró una adecuada consistencia interna total de .79 y tres factores que explicaron el $40.65 \%$ de la varianza. Se concluye que la versión mexicana de dicha prueba posee propiedades psicométricas adecuadas para la medición de positividad en universitarios mexicanos.

Palabras clave: adaptación, estudiantes, positividad, internet, validez y confiabilidad.

\section{ABSTRACT}

The purpose of this study was to translate, adapt, validate and determine the reliability of self test positivity (Fredrickson, 2009) on the Internet. To do this, the instrument was translated into Spanish, cultural relevance was corroborated by cognitive laboratories and tested in writing, and online college students. We determined the factor structure and reliability on the Internet in a nonrandom sample consists of 383 students from the School of Nursing and Obstetrics at the UNAM. The analysis showed a total internal consistency .79 and three factors that accounted for $40.65 \%$ of the variance. We conclude that the Mexican version of the test has adequate psychometric properties for measuring positivity in Mexican university.

Keywords: adaptation; students; positivity, internet, validity and reliability.

\footnotetext{
" Recibido: 1 de noviembre, 2012 - Aceptado: 25 de marzo, 2013

Correspondencia: Laura Graciela Cortina Guzmán, Av. Universidad 3000, C. P. 04510, México, D. F.

e-mail: cortinaguzman@gmail.com, tel. (52)(044) 5529470443.
} 


\section{INTRODUCCIÓN}

Por mucho tiempo las emociones negativas ${ }^{1}$ han tenido presencia en la investigación psicológica, procurando explicar su relación con lo que altera la salud mental o propicia la enfermedad (Fredrickson, 2003). Sin embargo, en los últimos años, los psicólogos positivos sustentan científicamente que las emociones positivas $^{2}$ son elementos básicos para el funcionamiento óptimo (Lynley \& Joshep, 2004) y el bienestar (Ryan \& Deci, 2000) de las personas. Este renovado interés sostiene que las emociones positivas no sólo hacen sentir bien (Compton, 2005), sino que cumplen una función adaptativa y son un medio para alcanzar el bienestar a largo plazo (Fredrickson, 2001; Seligman \& Csikszentmihalyi, 2002; Vázquez, Hervás \& Ho, 2006).

En cuanto a la función adaptativa, la literatura señala por un lado, que las emociones negativas son soluciones eficientes a problemas recurrentes e inmediatos (Fredrickson, 2004). Por ejemplo, ante un sentimiento de miedo surge la necesidad de huir por lo que el organismo se prepara para movilizarse. A esta reacción se le conoce como tendencia especifica de acción, la cual permite actuar con rapidez y decisión como forma natural de selección humana y universal (Tooby \& Cosmides, 1990; Oatley \& Jenkins, 1996).

Por otra parte, muestra que las emociones positivas, a pesar de parecer desvinculadas de la acción inmediata, cumplen otra función no menos importante relacionada con el comportamiento adaptativo: el pensamiento-acción; por ejemplo, al vivir la alegría surge la necesidad de jugar, explorar, manifestar y compartir esa emoción con otros, incluso, bajo ese estado se manifiesta la creatividad (Isen, 1999). De este modo ambas emociones involucran cambios coordinados de pensamientos, acciones y respuestas fisiológicas que conducen a la adaptación humana (Fredrickson, 1998; Fredrickson \& Branigan, 2005).

No obstante, además de la evidencia con respecto al valor adaptativo del afecto, los estudios señalan que el conjunto de pensamientos y acciones originados particularmente por los buenos sentimientos facilita el

${ }^{1}$ Las emociones negativas son sensaciones desagradables que concentran la atención en la toma de decisiones para lo que se percibe como una amenaza (Carr, 2007).

${ }^{2}$ Las emociones positivas son sensaciones agradables que facilitan la atención sobre el entorno físico y social de forma consciente (Carr, 2007). desarrollo de recursos físicos, sociales y psicológicos, los cuales, a su vez, contribuyen de manera significativa a la resiliencia, la salud y el bienestar (Forgas, 2002; Fredrickson \& Joiner, 2002; Waugh \& Fredrickson, 2006; Aspinwall, 2001; Keyes, 2002; Lyumbormisky, King \& Diener, 2005; Pekrun, Goetz, Titz \& Perry, 2002; Tugade \& Fredrickson, 2007).

Una variedad de resultados empíricos apoyan estos fundamentos, demostrando que en relación con la salud, las emociones positivas inducidas reducen la probabilidad de enfermar evitando la incapacidad y el deterioro físico, lo que facilita la longevidad con menores condiciones mórbidas, inmunológicas y cardiacas. Además, ayudan a la recuperación fisiológica provocada por las emociones negativas y fortalecen al individuo frente a la depresión (Cohen, Doyle, Turner, Alper \& Snoker, 2003; Danner, Snowdon \& Friesen, 2001; Fredrickson, Mancuso, Branigan \& Tugade 2000; Fredrickson, Tugade, Waugh \& Larkin, 2003; Gable \& Impett, 2003; Keyes, 2007; Ostir et al., 2002; Ostir, Markides, Black \& Goodwin, 2000; Pressman \& Cohen, 2005).

Claramente esto sugiere que las emociones positivas son el polo opuesto de las negativas, y el único componente para proteger la salud; sin embargo, los psicólogos positivos sostienen que son una parte de la estructura afectiva de la vida de una persona y un extremo de la misma dimensión, cuyo balance asociado a pensamientos y actitudes, en un momento, dado determina la salud mental positiva (Fredrickson \& Losada, 2005; Keyes, 2002, 2007). En apoyo a esta idea Fredrickson, Tugade, et al. (2003) han demostrado que vivir emociones tanto positivas como negativas es inevitable y además útil; por ejemplo, atravesar por eventos adversos como el duelo, puede privar a las personas de ejercer roles productivos y afectuosos; sin embargo, proporcionar un significado positivo a la circunstancia también puede impulsar a las personas a sentirse bien, a enfrentar la adversidad para salir adelante y lograr la satisfacción en la vida (Fredrickson \& Losada, 2005).

En concreto, esta perspectiva sostiene que en contraste con el beneficio adaptativo de las emociones negativas (inmediato en situaciones de amenaza), los sentimientos positivos construyen los recursos cognitivos, conductuales, físicos y sociales que llevan indirectamente, y a largo plazo, a la adaptación, asumiendo 
individuos más sanos y más integrados a la sociedad (Fredrickson \& Losada, 2005; Keyes, 2002, 2007).

A pesar del esfuerzo de los investigadores para esclarecer el papel que juegan las emociones positivas en el óptimo funcionamiento psicosocial individual y colectivo, la psicología necesita más estudios que apoyen el uso de estas emociones y sirvan de parámetro para mejorar la vida de las personas. En este sentido, resulta de especial interés apuntalar el desarrollo de instrumentos basados en la detección de emociones positivas para ser promovidas y encaminadas a soslayar las dificultades que han tenido los científicos al considerar la salud mental en términos positivos.

Desde ese punto de vista ha sido de suma importancia el diseño de herramientas fiables para establecer el estado emocional de los individuos de manera integrada y estandarizada en conjunto con los diversos aspectos de la salud. En el presente trabajo se hace referencia a algunas características de los estudios que han dado origen al instrumento de nuestro interés (ver tabla 1).

Tabla 1. Estudios comprometidos en el desarrollo de la prueba de positividad

\begin{tabular}{|c|c|c|c|c|}
\hline Estudio & Escala & Descripción & Objetivo & Alfa de Cronbach \\
\hline Izard 1977 & $\begin{array}{l}\text { Escala diferencial } \\
\text { de emociones } \\
\text { (DES) }\end{array}$ & $\begin{array}{l}10 \text { emociones básicas y su } \\
\text { combinación con tres adjetivos } \\
\text { relacionados para cada una. } \\
\text { Intensidad emocional en una } \\
\text { escala de cinco puntos que van de } \\
\text { nada a extremadamente. }\end{array}$ & $\begin{array}{l}\text { Evaluar experiencia } \\
\text { individual de las } \\
\text { emociones en una } \\
\text { situación dada. }\end{array}$ & $\begin{array}{l}\text { Alegría } .76 \\
\text { Sorpresa.87 } \\
\text { Ira .75 } \\
\text { Disgusto .85 } \\
\text { Desprecio .68 } \\
\text { Vergüenza .73 } \\
\text { Culpa .68 } \\
\text { Miedo .78 } \\
\text { Interés .83 } \\
\text { Tristeza } .77\end{array}$ \\
\hline $\begin{array}{l}\text { Fredrickson, Tugade, } \\
\text { Waugh y Larkin } \\
\text { (2003) }\end{array}$ & DES-M & $\begin{array}{l}\text { Modificación del DES. } \\
20 \text { reactivos. Dos dimensiones } \\
\text { (positiva, negativa). Frecuencia } \\
\text { emocional en una escala de cinco } \\
\text { puntos que van de nunca a la } \\
\text { mayor parte del tiempo. }\end{array}$ & $\begin{array}{l}\text { Probar el efecto } \\
\text { protector de las } \\
\text { emociones positivas } \\
\text { ante la depresión y } \\
\text { eventos adversos. }\end{array}$ & $\begin{array}{l}\text { Escala positiva } .79 \\
\text { Escala negativa } .69\end{array}$ \\
\hline $\begin{array}{l}\text { Fredrickson y Losada } \\
\text { (2005) }\end{array}$ & DES $-M$ & $\begin{array}{l}20 \text { reactivos } \\
\text { Intensidad emocional en una } \\
\text { escala de cinco puntos que van de } \\
\text { nada a extremadamente. }\end{array}$ & $\begin{array}{l}\text { Establecer la relación } \\
\text { entre el afecto } \\
\text { positivo y la salud } \\
\text { mental floreciente. }\end{array}$ & NM \\
\hline $\begin{array}{l}\text { Waugh y Fredrickson } \\
\text { (2006) }\end{array}$ & DES -M & $\begin{array}{l}20 \text { reactivos } \\
\text { Frecuencia e intensidad en una } \\
\text { escala de cinco puntos (nada } \\
\text { a extremadamente, nunca a la } \\
\text { mayor parte del tiempo). }\end{array}$ & $\begin{array}{l}\text { Comprobar la } \\
\text { relación de altas } \\
\text { tasas de positividad } \\
\text { con la formación de } \\
\text { relaciones sociales } \\
\text { en tres muestras y } \\
\text { tiempos diferentes. }\end{array}$ & $\begin{array}{l}\text { Escala positiva } \\
\text { Tiempo } 0.79 \\
\text { Tiempo } 1.79 \\
\text { Tiempo } 2.89 \\
\text { Escala negativa } \\
\text { Tiempo } 0.80 \\
\text { Tiempo } 1.84 \\
\text { Tiempo } 2.82\end{array}$ \\
\hline Fredrickson (2009) & $\begin{array}{l}\text { Prueba de } \\
\text { Positividad }\end{array}$ & $\begin{array}{l}20 \text { reactivos divididos } \\
\text { equitativamente en dos } \\
\text { dimensiones: positiva y negativa. } \\
\text { Intensidad en escala de cinco } \\
\text { puntos que van de nada a } \\
\text { extremadamente. }\end{array}$ & $\begin{array}{l}\text { Medir la proporción } \\
\text { de afecto positivo y } \\
\text { negativo en relación } \\
\text { a la salud mental. }\end{array}$ & NM \\
\hline
\end{tabular}


Originalmente, Izard (1977) diseñó la escala diferencial de emociones (DES, por las siglas en inglés de Differential Emotions Escale) para conocer la intensidad de 10 emociones básicas. ${ }^{3}$ La estructura contiene un trío de palabras relacionadas para cada emoción, por ejemplo, interés: atento/concentrado/alerta. Tal conjunto de adjetivos, actualmente, conforma las distintas versiones del DES con instrucciones particulares y escalas de múltiples puntos para medir tanto sentimientos actuales como rasgos afectivos (experiencia emocional a través del tiempo) (Izard, 1977).

Más adelante, Fredrickson, Tugade, et al. (2003), emplearon el DEs; agregaron ocho emociones positivas (diversión, asombro, contento, gratitud, esperanza, amor, orgullo, deseo sexual) a las ya existentes (alegría e interés) y conservaron las ocho emociones negativas (ira, tristeza, miedo, disgusto, desprecio, vergüenza, culpa, timidez) propuestas por Izard en 1977. Los autores concluyeron que las emociones positivas protegen de la depresión y ayudan a las personas resilientes a progresar después de experimentar dificultades en la vida.

Posteriormente, Fredrickson y Losada (2005), con base en la teoría de ampliación y construcción de las emociones positivas y el modelo dinámico no lineal (Fredrickson, 1998; Losada, 1999) utilizaron el DEs para calcular y comparar la proporción de afecto positivo y negativo; durante 28 días evaluaron en un sitio de internet el grado en que cada emoción positiva (diversión, asombro, compasión, gratitud, esperanza, interés, amor, orgullo, deseo sexual, alegría, contento) fue experimentada por lo menos moderadamente $(\geq 2)$ y cada emoción negativa (ira, desprecio, disgusto, pena, miedo, culpa, tristeza, vergüenza) al menos un poco $(\geq 1)$. El resultado determinó que la relación de afecto positivo con la salud mental floreciente está en y por encima de una proporción de 3:1 (razón de positividad).

En otro momento, Waugh y Fredrickson (2006) aplicaron el DEs en internet con una escala de 11 emociones positivas (asombro, diversión, alegría, agradecimiento, contento, optimismo, interés, amor, orgullo, simpatía, sorpresa) y una escala de ocho emociones negativas (enojo, desprecio, vergüenza, disgusto, triste-

\footnotetext{
${ }^{3}$ Una emoción básica es un conjunto de componentes neurales y corporales innatos de expresión universal, y de sentimientos/motivaciones no conscientes que convergen en funciones biológicas y sociales, esenciales para la evolución y la adaptación (Izard, 2007).
}

za, culpa, miedo, pena) a tres muestras en tres tiempos respectivamente $(0=3$ semanas, $1=$ una semana, $2=$ un mes (28 días). Recurrieron a la razón de positividad y probaron que ésta predice de manera significativa la formación de una relación social y la comprensión hacia otras personas.

En suma, estos trabajos han dado origen a la medida propuesta por Fredrickson (2009) para conocer la proporción de emociones positivas y negativas relacionada con la salud mental floreciente o el estado de languidez ${ }^{4}$ de una persona: la auto prueba de positividad (Positivity Self Test). La prueba se describe más adelante, en el apartado de instrumento.

$\mathrm{Al}$ respecto, puede observarse la normalización establecida para universitarios estadounidenses con medidas confiables y válidas. No obstante, es de suma importancia reconocer que la respuesta emocional se manifiesta de acuerdo con el contexto social y cultural.

Es así que el objetivo de este trabajo fue traducir y adaptar al español el Positivity Self Test para llevar a cabo la validación del instrumento en una muestra universitaria mexicana. Lo anterior con el fin de contar con una herramienta para posteriores investigaciones relacionadas con la positividad.

\section{MÉTODO}

\section{Participantes}

El proceso de adaptación incluyó a 88 estudiantes de ambos sexos inscritos en la Escuela Nacional de Enfermería y Obstetricia de la Universidad Nacional Autónoma de México. De los cuales, 10 fueron examinados (50\% mujeres, $50 \%$ hombres) con el método de laboratorio cognitivo, 70 participaron (58 mujeres, 12 hombres) en una aplicación escrita y ocho más comprobaron el uso del instrumento en internet. Finalmente, para el proceso de validación se conformó una muestra no probabilística intencional con 383 estudiantes universitarios ( $84 \%$ mujeres, $16 \%$ hombres) entre 17 y 43 años con edad promedio de $20.69 \pm 3.70$

\footnotetext{
${ }^{4}$ El florecimiento es un estado de salud mental, caracterizado por altos niveles de bienestar subjetivo, psicológico y social, asociados con una alta proporción de afectividad positiva. Por su parte, en la languidez, aunque también se le considera un estado de salud mental, se combinan bajos niveles de bienestar subjetivo, psicológico y social, asociados con mayor presencia de afecto negativo (Keyes, 2002, 2005, 2007; Ryan \& Deci, 2000; Westerhof \& Keyes, 2010).
} 
años inscritos en el primer (45\%), tercero (22\%), quinto $(28 \%)$ y séptimo (4\%) semestres de la carrera y escuela antes mencionada. Del total de la muestra, $89 \%$ era soltero(a) y $69 \%$ católico(a) al momento de este estudio (ver tabla 2).

Tabla 2. Características sociodemográficas de la muestra $(n=383)$

\begin{tabular}{l|l|l}
\hline Variables & $\mathbf{f}$ & $\%$ \\
\hline
\end{tabular}

Edad

\begin{tabular}{l|c|c}
\hline $17-21$ & 286 & 74.7 \\
\hline $22-25$ & 78 & 20.2 \\
\hline $27-36$ & 14 & 3.7 \\
\hline 40 y más & 5 & 1.3 \\
\hline
\end{tabular}

Estado civil

\begin{tabular}{l|c|c}
\hline Soltero(a) & 340 & 88.8 \\
\hline Unión libre & 16 & 4.2 \\
\hline Casado(a) & 17 & 4.4 \\
\hline Separado(a) & 7 & 1.8 \\
\hline Divorciado(a) & 1 & .3 \\
\hline Viudo(a) & 2 & .5 \\
\hline
\end{tabular}

\section{Religión}

\begin{tabular}{l|c|c}
\hline Católico(a) & 266 & 69.5 \\
\hline Creyente & 49 & 12.8 \\
\hline Ninguna & 41 & 10.7 \\
\hline Cristiano(a) & 26 & 6.8 \\
\hline Otra & 1 & .3 \\
\hline
\end{tabular}

\section{Instrumento}

La versión del Positivity Self Test (Fredrickson, 2009) está compuesta por 20 reactivos que evalúan la experiencia emocional con base en la proporción entre el afecto positivo y el negativo. Cada reactivo consiste en un trío de palabras que comparten semejanza (divertido, juguetón, simplón), al que los sujetos responden de acuerdo con el grado en que las experimentan en cinco puntos de anclaje, que van del 0 al 4 , los cuales significan $0=$ Nada, $1=$ Un poco, $2=$ De forma moderada, $3=$ Mucho y 4=Muchísimo. La calificación se obtiene de la división entre el número total de reactivos positivos experimentados por lo menos moderadamente $(\geq 2)$ y el número total de reactivos negativos sentidos al menos un poco $(\geq 1)$. El resultado es indicativo de florecimiento si es igual o mayor a una razón de 3.1:1, y de languidez si se encuentra por debajo de este. La implementación de esta herramienta abarca tanto los escenarios físicos como virtuales (internet). Como se describe más adelante, ambas modalidades fueron utilizadas en el presente estudio.

\section{Procedimiento}

Inicialmente, el instrumento fue traducido al español y retraducido al inglés por dos pares de expertos traductores en ambos idiomas. Después, esta versión fue sometida al conocimiento de otros dos traductores para una última traducción al español. Más tarde un grupo de psicólogos acordó la pertinencia cultural. Con la versión resultante se entrevistó a 10 alumnos de forma individual mediante el método de laboratorio cognitivo concurrente ${ }^{5}$; se exploró el significado de conceptos clave (p. e. "enojado", "asombrado", "orgulloso", entre otros), las alternativas para expresar el mismo concepto y las formas de explicarlo a alguien más. La información fue discutida y concluida por psicólogos para la redacción final. En este sentido, el laboratorio cognitivo corroboró la calidad en la interpretación de las diferentes culturas involucradas (Nolin \& Chandler, 1996).

\footnotetext{
${ }^{5}$ Los laboratorios cognitivos pueden aplicarse de manera individual o grupal para construir o adaptar un instrumento. Se clasifican en dos grupos: el primero comprende métodos concurrentes, los cuales consisten en proporcionar en un momento único información, articular el significado y/o recordar un proceso mental; el segundo comprende los métodos retardados, donde las tareas cognitivas se llevan a cabo después de haber respondido la pregunta (Nolin \& Chandler,1996).
} 
Con la finalidad de asegurar la comprensión del instrumento se solicitó el consentimiento de profesores para ceder sus horarios de clase. Después, 70 estudiantes completaron de forma individual una impresión en papel de la prueba y un cuestionario de evaluación sobre la misma. Al final, se analizaron todos los comentarios, eliminando las confusiones en instrucciones, reactivos y ejemplos.

Al término de la aplicación escrita se probó la viabilidad del instrumento para ser respondido en internet. Al principio se contrató un sitio seguro de internet y se adecuó el contenido de la prueba a las características del mismo. Posteriormente se estableció contacto con los alumnos en el horario escolar y se les solicitó su colaboración. Después de informarles el propósito del estudio, ocho participantes proporcionaron un correo electrónico mediante el cual se les comunicó el vínculo de acceso a la prueba y las instrucciones para responderla; se les pidió contestar a todas las preguntas con sinceridad, haciendo hincapié en la importancia de reenviar un correo electrónico con las ventajas e inconvenientes que experimentaron durante el proceso. El tiempo que llevó a los participantes responder en línea fue de 15 minutos, aproximadamente. Es importante mencionar que la página permaneció abierta las 24 horas del día y el acceso se pudo realizar desde cualquier equipo de cómputo con internet.

Por otro lado, la información en el sitio de internet se presentó en el siguiente orden: a) propósito del estudio, b) instrucciones de respuesta, c) cuenta de correo electrónico para resolver dudas, d) información sociodemográfica, e) instrumento y f) agradecimiento. Además, se dispuso del envío automático de la información a una base datos, una vez que el respondiente cumplió con el total de preguntas.

Por último, la versión definitiva se aplicó en internet (en las condiciones antes mencionadas) a 383 estudiantes que aceptaron ayudar a las investigadoras a conocer las emociones que experimentan los universitarios y a beneficiarse de un curso presencial de bienestar emocional ${ }^{6}$. Al concluir, se evalúo la validez y confiabilidad de dicho instrumento.

${ }^{6}$ El curso de bienestar emocional se proporcionó de forma gratuita a los participantes que completaron la medida de positividad; consistió en cinco sesiones de cuatro horas cada una con temas reportados en la investigación de la psicología positiva.
Antes de las entrevistas, aplicaciones escritas y vía internet, los directivos de la institución educativa y los participantes fueron notificados del consentimiento informado con la explicación del objetivo del estudio, las condiciones de voluntariedad, confidencialidad y anonimato.

\section{RESULTADOS}

\section{Traducción y adaptación al contexto cultural}

Se realizó la traducción y retraducción de la versión original por expertos traductores en ambos idiomas y en psicología clínica. La versión final resultó del acuerdo entre investigadores en psicología con especialidad en psicometría. Se determinó el empleo de los términos más comprensibles y adecuados a la cultura con el método de laboratorio cognitivo, por el cual se comprobó la constancia de sinonimia entre las palabras que componen cada reactivo, por lo que se realizaron modificaciones. Se eliminaron los términos ambiguos y se conservaron los que mejor expresaron a la emoción principal.

\section{Análisis de reactivos}

La aplicación de la prueba $t$ de Student demostró el adecuado poder de discriminación de los reactivos $(\mathrm{p}=.000)$.

\section{Validez factorial}

La evaluación de la matriz de datos empleando el índice de adecuación de la muestra Kaiser-Meyer-Olkin (KMO) con valor de .854 y la Prueba de Esfericidad de Bartlett situada en $x^{2}=2799.705, \mathrm{p}=.000$ indicaron la pertinencia para realizar el análisis factorial. Para éste se consideraron los reactivos con pesaje factorial $\geq .40$ $\mathrm{y}$ se definieron como factores a partir de tres reactivos (ver tabla 3 ).

El análisis factorial exploratorio mediante el método de componentes principales con rotación Varimax arrojó tres factores que integraron 20 reactivos, explicando $40.65 \%$ de la varianza total.

Los resultados del análisis factorial determinaron "renombrar" las agrupaciones para dar lugar a una lectura válida y congruente del constructo.

Los factores obtenidos fueron: 1) componente emocional positivo (10 reactivos) con disposición a sentimientos positivos (diversión, asombro, agradecimiento, 
Tabla 3. Estructura factorial de la auto prueba de positividad

\begin{tabular}{|c|c|c|c|}
\hline \multirow[t]{2}{*}{ Variables } & \multicolumn{3}{|c|}{ Factor } \\
\hline & 1 & 2 & 3 \\
\hline ¿Qué tan inspirado(a) te sentiste el día de ayer? & .81 & & \\
\hline ¿Qué tan interesado(a) te sentiste el día de ayer? & .74 & & \\
\hline ¿Qué tan esperanzado(a) te sentiste el día de ayer? & .73 & & \\
\hline ¿Qué tan orgulloso(a) te sentiste el día de ayer? & .61 & & \\
\hline ¿Qué tan alegre te sentiste ayer? & .55 & & \\
\hline ¿Qué tan divertido(a) te sentiste el día de ayer? & .54 & & \\
\hline ¿Qué tan agradecido(a) te sentiste el día de ayer? & .54 & & \\
\hline ¿Qué tan asombrado(a) te sentiste el día de ayer? & .53 & & \\
\hline ¿Qué tan amoroso(a) te sentiste el día de ayer? & .52 & & \\
\hline ¿Qué tan sereno(a) te sentiste el día de ayer? & .47 & & \\
\hline ¿Qué tan estresado(a) te sentiste el día de ayer? & & .71 & \\
\hline ¿Qué tan fastidiado(a) te sentiste el día de ayer? & & .63 & \\
\hline ¿Qué tan enojado(a) te sentiste el día de ayer? & & .57 & \\
\hline ¿Qué tan triste te sentiste el día de ayer? & & .51 & \\
\hline ¿Qué tan rencoroso(a) te sentiste el día de ayer? & & .43 & \\
\hline ¿Qué tan ofensivo(a) te sentiste el día de ayer? & & .40 & \\
\hline ¿Qué tan avergonzado(a) te sentiste el día de ayer? & & & 69 \\
\hline ¿Qué tan apenado(a) - o tímido(a) te sentiste el día de ayer? & & & .58 \\
\hline ¿Qué tan culpable te sentiste el día de ayer? & & & .57 \\
\hline ¿Qué tan asustado(a) -o inseguro(a) te sentiste el día de ayer? & & & .55 \\
\hline Coeficientes alfa $=$ & .86 & .76 & .73 \\
\hline
\end{tabular}


esperanza, inspiración, interés, alegría, amor, orgullo y serenidad); 2) vivencia emocional negativa (seis reactivos) con sensación de malestar, desesperación y cansancio (enojo, ofensa, rencor, tristeza, fastidio, estrés); 3) percepción emocional negativa (cuatro reactivos) con percepción de incertidumbre e incomodidad (asustado-inseguro, pena-timidez, vergüenza, culpa).

Estos datos representan una forma de validez de constructo (Kerlinger \& Lee, 2002) y develan el constructo subyacente del conjunto de variables observadas y diferenciadas bajo el cual se agrupan los reactivos de la versión mexicana del instrumento (ver Anexo).

\section{Confiabilidad}

La evaluación de la consistencia interna utilizando el método alfa de Cronbach determinó el adecuado índice de fiabilidad global de .795 y de .899 para el componente emocional positivo, de .768 para vivencia emocional negativa y de .736 para percepción emocional negativa.

\section{DISCUSIÓN}

Desde un punto de vista sustantivo, los resultados responden al propósito de contar con un instrumento válido y fiable basado en las características de una muestra estudiantil mexicana.

El proceso de adaptación tuvo hallazgos interesantes, en los cuales se observa una amplia gama de adjetivos para designar emociones que reflejan la multiplicidad de los usos del lenguaje al interpretar la presencia emocional. En este sentido, la exploración de un número mayor de adjetivos puede ampliar el conocimiento sobre la expresión emocional con fundamento en la cultura. No se encontró otro estudio que haya traducido y utilizado este instrumento en México.

Por su parte, el proceso de validez comprendió un análisis factorial exploratorio de componentes principales y rotación Varimax, en el cual las dos dimensiones (positiva y negativa) documentadas por la autora se descomponen en tres factores, aislando una parte de los reactivos relacionados con la dimensión negativa. Por ello, la agrupación resultante se precisó de la siguiente manera: componente emocional positivo: resultó ser el conjunto de emociones más representativo para esta muestra y directamente afín con el marco teórico. Concentró el total de emociones agradables, cuyo denominador común es el placer (Lucas, Clark, Georgellis \& Diener, 2003).

Es posible que esta clara identificación de los descriptores de afecto positivo resida en la propensión de las personas a adaptarse con mayor facilidad a lo agradable a pesar de los acontecimientos negativos de la vida (Brickman, Coates \& Janoff-Bulman, 1978). No obstante, Diener, Lucas y Scollon (2006) sostienen que existe una tendencia de adaptación más fuerte para lo negativo que para lo positivo. A pesar de que ambas posturas son susceptibles de futuras comprobaciones, se ha documentado que el cúmulo de emociones positivas requiere de una postura intencional para alcanzar el bienestar (Fredrickson, Coffey, Pek, Cohn \& Finkel, 2008; Lyubomirsky, Sheldon \& Schkade, 2005).

Vivencia emocional negativa: se formó de emociones negativas (enojo, ofensa, rencor, tristeza, fastidio, estrés) generadas por las exigencias sociales. Esta composición quizás aborda la necesidad de cumplir con las obligaciones escolares y familiares asumidas por esta muestra en esta etapa del desarrollo. Estas emociones ponen de manifiesto lo reportado en la literatura con respecto al efecto que tienen para responder de manera concreta (tendencias especificas de acción) a las condiciones ambientales evaluadas como amenazantes (Frijda, 1986; Lerner \& Keltner, 2000).

Percepción emocional negativa: conjugó emociones relacionadas con la evaluación o retroalimentación externa. Este conjunto de reactivos posiblemente se sustente en la interpretación acerca de los significados que otros expresan en relación con determinadas conductas o circunstancias. Esta idea concuerda con los postulados de Ackerman, Abe e Izard (1998), quienes señalan que las emociones de vergüenza, culpa y desprecio aparecen cuando se percibe que los otros son agentes causales o desencadenantes de una evaluación.

Es importante destacar que las diferencias halladas con respecto a la estructura factorial no invalidan el instrumento, por el contrario, expresan la multidimensionalidad emocional que representa este marco sociocultural. En este sentido, se evidencia la necesidad de crear nuevas preguntas de investigación para conocer los procesos que se articulan en la experiencia emocional.

Por otra parte, la consistencia interna resultó ser concordante con lo documentado, así como el alfa 
obtenido para las subescalas es muy similar en los estudios realizados (Fredrickson, Tugade et al., 2003; Fredrickson \& Losada, 2005; Waugh \& Fredrickson, 2006). Con esto se comprueba que la versión mexicana posee índices de consistencia interna adecuados.

En lo que respecta a las limitaciones del estudio, resalta el hecho de que la aplicación se sujetó a las necesidades del objetivo por lo que no se consideraron los reportes acerca de la proporción de positividad. Si bien estos datos son relevantes y sería recomendable indagarlos, Fredrickson (2009) aconseja mantener medidas constantes (por lo menos durante dos semanas) para incrementar la certeza sobre aquellas emociones que podrían propiciar un buen estado de salud. Esto podría estudiarse con aplicaciones subsecuentes y re- visiones constantes sobre la fluctuación emocional que lleven a diluir la percepción básica de la que es susceptible una única medición, y cuya tendencia se encuentra frecuentemente en las opciones de respuesta tipo Likert (Sierra, 1994; Payne, Cheng, Govorun \& Stewart, 2005).

En cuanto a las aportaciones de este trabajo se enfatiza la contribución a la investigación en el contexto de la psicología positiva y el uso del internet para medir de forma sencilla emociones.

Para finalizar, el instrumento de positividad en su versión en español y aplicado en universitarios mexicanos muestra propiedades psicométricas adecuadas para considerarse una medida válida y confiable. 


\section{REFERENCIAS}

Ackerman, B. P., Abe, J. A. e Izard, C. (1988). Differential emotions theory and emotional development: Mindful of modularity. En M. Mascolo y S. Griffin (Eds.), What develops in emotional development? Emotions, Personality and Psychotherapy (pp.85-106). NY: Plenum Press.

Aspinwall, L. G. (2001). Dealing with adversity: Selfregulation, coping, adaptation, and health. En A. Teasser y N. Schwarz (Eds.), The Black-well handbook of social psychology. Intrapersonal processes (pp. 159164). Malden, MA: Blackwell.

Brickman, P., Coates, D. \& Janoff-Bulman, R. (1978). Lottery winners and accident victims: Is happiness relative? Journal of Personality and Social Psychology, 36, 917-927.

Carr, A. (2007). Psicología positiva. Barcelona, España: Paidós Ibérica.

Cohen, S., Doyle, W., Turner, R., Alper, C. \& Skoner (2003). Emotional style and susceptibility to the common cold. Psychosomatic Style, 65, 652-657.

Compton, W. (2005). Introduction to Positive Psychology. Belmont, CA: Thomson Wadsworth Press.

Danner, D., Snowdon, D. \& Friesen, W. (2001). Positive emotions in early life and longevity: Findings from the nun study. Journal of Personality and Social Psychology, 80, 804-813.

Diener, E., Lucas, R. E. \& Scollon, C. N. (2006). Beyond the hedonic treadmill: Revising the adaptation theory of well-being. American Psychologist, 61, 305-314.

Forgas, J. P. (2002). Feeling and doing: Affective influences on interpersonal behavior. Psychological Inquiry, 13, 1-28.

Fredrickson, B. L. (1998). What good are positive emotions? Review of General Psychology, 2, 300-319.

Fredrickson, B. L. (2001). The role of positive emotions in positive psychology: The broaden and build theory of positive emotions. American psychology, 56(3), 218-226.

Fredrickson, B. L. (2003). The value of positive emotions. American Scientist, 91, 330-335.

Fredrickson, B. L. (2004). The broaden and build of positive emotions. The Royal Society, 359, 1367-137.

Fredrickson, B. L. (2009). Positivity (1a. ed.). NY: Crown Publishers.
Fredrickson, B. L. \& Branigan, C. (2005). Positive emotions broaden the scope of attention and thought-action repertories. Cognition and Emotion, 19(3), 313-332.

Fredrickson, B. L., Coffey, K. A. Pek, J., Cohn, M. A. \& Finkel, S. M. (2008). Open Hearts Build Lives: Positive Emotions, Induced Through Loving-Kindness Meditation, Build Consequential Personal Resources. Journal of Personality and Social Psychology, 95(5), 1045-1062.

Fredrickson, B. L., Mancuso, R., Branigan, C. \& Tugade, M. (2000). The Undoing Effect of Positive Emotions. Motivation and Emotion, 24(4), 237-257.

Fredrickson, B. L., Tugade, M. M., Waugh, C. E., \& Larkin, G. (2003). What good are positive emotions in crises? A prospective study of resilience and emotions following the terrorist attacks on the United States on September 11th, 2001. Journal of Personality and Social Psychology, 84, 365-376.

Fredrickson, B. L. \& Joiner, T. (2002). Positive emotions trigger upward spirals toward emotional well-being. Psychological Science, 13, 172-175.

Fredrickson, B. L. \& Losada, M. (2005). Positive Affect and the Complex Dynamics of Human Flourishing. American Psychologist, 60(7), 678-686.

Frijda, N. H. (1986). The emotions. Cambridge, Inglaterra: Cambridge University Press.

Gable, S. e Impett, E. (2003). What do you when things go right? The intrapersonal and interpersonal benefits of sharing positive events. Journal of Personality and Social Psychology, 1-30.

Isen, A. M. (1999). On the relationship between affect and creative problem solving. En S. Russ (Eds.), Affect, creative experience and psychological adjustment (pp. 3-17). Filadelfia: Taylor y Francis.

Izard, C. E. (1977). Human emotions. Ny: Plenum Press.

Izard, C. E. (2007). Basic emotions, natural kinds, emotion schemas, and a new paradigm. Perspectives on Psychological Science, 2(3), 260-280.

Kerlinger, F. \& Lee H. (2002). Investigación del comportamiento. México: McGraw Hill.

Keyes, C. L. M. (2002). The Mental Health Continuum: From Languishing to Flourishing in Life. Journal of Health and Social Behavior, 43, 207-222.

Keyes, C. L. M. (2005). Mental illness and/or mental health? Investigating axioms of the complete state 
model of health. Journal of Consulting and Clinical Psychology, 73, 539-548.

Keyes, C. L. M. (2007). Promoting and protecting mental health as flourishing. A complementary strategy for improving national mental health. American Psychologist, 62(2), 95-108.

Lerner, J. S. \& Keltner, D. (2000). Beyond valence: Toward a model of emotion-specific influences on judgment and choice. Cognition and Emotion, 14(4), 473-493.

Linley, A. \& Joseph, S. (2004). Applied Positive Psychology: A New Perspective for Professional Practice. En S. Joseph y P. A. Linley (Eds.), Positive Psychology In Practice. (pp.3-12). New Jersey: Hoboken John Wiley and Sons.

Losada, M. (1999). The complex dynamics of high performance teams. Mathematical and Computer Modelling, 30(9-10), 179-192.

Lucas, R. E., Clark, A. E., Georgellis, Y. \& Diener, E. (2003). Reexamining adaptation and the set point model of happiness: Reactions to changes in marital status. Journal of Personality and Social Psychology, 84, 527-539.

Lyubomirsky, S., King, L. \& Diener, E. (2005). The benefits of frequent positive affect: Does happiness lead to success? Psychological Bulletin, 131(6), 803-855.

Lyubomirsky, S., Sheldon, K. S. \& Schkade, D. (2005). Pursuing happiness: The architecture of sustainable change. Review of General Psychology, 9(2), 111-131.

Nolin, M. J. \& Chandler, K. (1996). Use of cognitive laboratories and recorded interviews in the National Household Education Survey. Washington: US Department of Education, National Center for Education Statistics.

Oatley, K. \& Jenkins, J. M. (1996). Understanding emotions. Cambridge, MA: Blackwell.

Ostir, G., Goodwin, J., Markides, K., Balfour, J., Ottenbacher, K. \& Guralnik, J. (2002). Differential effects of premorbid physical and emotional health on recovery from acute illness. Journal of the American Geriatrics Society, 50(4), 713-718.
Ostir, G., Markides, K., Black, S. \& Goodwin, J. (2000). Emotional well-being predicts subsequent functional independence and survival. Journal of the American independence and survival, 48, 473-478.

Payne, B. K., Cheng, C. M., Govorun, O., \& Stewart, B. D. (2005). An inkblot for attitudes: Affect misattribution as implicit measurement. Journal of Personality and Social Psychology, 89, 277-293.

Pekrun R., Goetz, T., Titz, W. \& Perry, R. (2002). Academic emotions in students' Self-regulated learning and achievement: A program of qualitative and quantitative research. Educational Psychologist, 2(37), 91-105.

Pressman, S. \& Cohen, S. (2005). Does positive affect influence health? Psychological Bulletin, 131(6), 925-971.

Ryan, R. \& Deci, E. (2000). Self-Determination theory and facilitation of intrinsic motivation, social development, and Well-Being. American Psychologist, 55(1), 68-78.

Seligman, M. \& Csikzentmilhayi, M. (2002). Positive Psychology. American Psychologist, 55(1), 5-14.

Sierra, R. (1994). Técnicas de investigación social. Teoría y ejercicios. Madrid: Paraninfo.

Tooby, J. \& Cosmides, L. (1990). The past explains the present: Emotional adaptations and the structure of ancestral environments. Ethology and Sociobiology, $11,375-424$.

Tugade, M. \& Fredrickson, B. L. (2007). Regulation of positive emotions: Regulation strategies that promote resilience. Journal of Happines Studies, 8, 311-333.

Vázquez, C., Hervás, G. \& Ho, S. (2006). Intervenciones clínicas basadas en la psicología positiva: fundamentos y aplicaciones. Psicología Conductual, 14(3), 401-432.

Waugh, C. E. \& Fredrickson, B. L. (2006). “Nice to know you": positive emotions, self-other overlap, and complex understanding in the formation of new relationships. Journal of Positive Psychology, 1(2), 93-106.

Westerhof, G. J. \& Keyes, C. L. M. (2010). Mental illness and mental health: The two continua model across the lifespan. Journal of Adult Development, 17(2), 110-119. 
ANEXO

\section{Autoprueba de positividad versión en español}

Las siguientes preguntas son acerca de diferentes emociones que las personas experimentan diariamente. Lee cada oración y marca el número que indica la fuerza con que sentiste cada emoción el día de ayer.

\begin{tabular}{|c|c|c|c|c|c|}
\hline & Nada & Un poco & $\begin{array}{l}\text { De forma } \\
\text { moderada }\end{array}$ & Mucho & Muchísimo \\
\hline ¿Qué tan divertido(a) te sentiste? & 0 & 1 & 2 & 3 & 4 \\
\hline ¿Qué tan enojado(a) te sentiste? & 0 & 1 & 2 & 3 & 4 \\
\hline ¿Qué tan avergonzado(a) te sentiste? & 0 & 1 & 2 & 3 & 4 \\
\hline ¿Qué tan asombrado(a) te sentiste? & 0 & 1 & 2 & 3 & 4 \\
\hline ¿Qué tan ofensivo(a) te sentiste? & 0 & 1 & 2 & 3 & 4 \\
\hline ¿Qué tan fastidiado(a) te sentiste? & 0 & 1 & 2 & 3 & 4 \\
\hline ¿Qué tan apenado(a) o tímido(a) te sentiste? & 0 & 1 & 2 & 3 & 4 \\
\hline ¿Qué tan agradecido(a) te sentiste? & 0 & 1 & 2 & 3 & 4 \\
\hline ¿Qué tan culpable te sentiste? & 0 & 1 & 2 & 3 & 4 \\
\hline ¿Qué tan rencoroso (a) te sentiste? & 0 & 1 & 2 & 3 & 4 \\
\hline ¿Qué tan esperanzado(a) te sentiste? & 0 & 1 & 2 & 3 & 4 \\
\hline ¿Qué tan inspirado(a) te sentiste? & 0 & 1 & 2 & 3 & 4 \\
\hline ¿Qué tan interesado (a) te sentiste? & 0 & 1 & 2 & 3 & 4 \\
\hline ¿Qué tan alegre te sentiste? & 0 & 1 & 2 & 3 & 4 \\
\hline ¿Qué tan amoroso(a) te sentiste? & 0 & 1 & 2 & 3 & 4 \\
\hline ¿Qué tan orgulloso(a) te sentiste? & 0 & 1 & 2 & 3 & 4 \\
\hline ¿Qué tan triste te sentiste? & 0 & 1 & 2 & 3 & 4 \\
\hline ¿Qué tan asustado (a) o inseguro(a) te sentiste? & 0 & 1 & 2 & 3 & 4 \\
\hline ¿Qué tan sereno(a) te sentiste? & 0 & 1 & 2 & 3 & 4 \\
\hline ¿Qué tan estresado(a) sentiste? & 0 & 1 & 2 & 3 & 4 \\
\hline
\end{tabular}

